\title{
Research on Video Evidence Volume Based on Cloud Storage
}

\author{
Feng $\mathrm{XU}^{*}$ \\ National Police University of China \\ Department of Forensic Science \& Technology \\ Shenyang, China
}

\begin{abstract}
With the development of the Ministry of public security's safety project, video surveillance detectors have been found in every corner of life, it provides an indispensable help to stabilize the public order and detection of criminal cases. In view of the existing problems of storage mode and monitoring video as direct evidence in litigation, this paper first analyzes the problems and challenges in the use of video evidence, and then constructs a video storage system based on cloud storage. Finally, according to the characteristics of monitoring video data involved in the case, the composition of video evidence volume is designed and some suggestions for the extraction and use of video images are proposed. The research of this paper makes the video surveillance storage develop to high capacity, high performance and intelligence. It is more reasonable and legitimate to use as evidence in judicial proceedings.
\end{abstract}

Keywords-Cloud storage; video evidence; evidence volume; judicial proceedings

\section{INTRODUCTION}

With the development of the Ministry of public security's safety project, the probe of video surveillance is widely distributed in important street, municipal key department, case multiple area, complex public place and mobile dense area, which provides an indispensable help for public security organs to carry out cases detection. Video surveillance technology is developing in the direction of high-definition, digitalization, networking and intellectualization [1-4].

With the continuous improvement of monitoring picture quality, the increase of video line and storage time, the storage capacity of the police monitoring system has increased ten times or even a hundred times, which seriously restricted the development of network video surveillance [5-7]. At the same time, the interpretation of people, cars, articles and events in the surveillance video can only provide clues for investigation and handling of cases in most cases. With the continuous improvement of judicial proceedings and the continuous improvement of criminal suspects' ability to counter reconnaissance, monitoring video as an independent evidence can't be directly in the process of litigation, when other auxiliary means (such as technical investigation, network detection, information) can't be directly identified as suspects.

The technical research project of the Ministry of public security under contract No. 2016JSYJC59 and national key research and development plan under contract No. 2017YFC0821000.
At present, there is no unified video evidence volume standard in the process of judicial proceedings. It is difficult to achieve the ideal application effect. These problems are the key problems to be solved urgently in the criminal technology department.

\section{THE PRESENT SITUATION OF VIDEO EVIDENCE}

In order to cope with the increasingly serious new situation of crime, the Ministry of public security put forward a new system of on-site investigation in the year 2014. This new mechanism is an important part of the new mechanism for combating crime, and is also an important prerequisite and basis for implementing a new mechanism to combat crime. "A director" is the chief responsibility of the chief public security officer of the county, which is responsible for the field investigation, and makes an overall plan to solve the problems of the personnel, funds, equipment and professional training, so as to ensure the work efficiency and quality of the field exploration. "Four Must" means that the work of on-site investigation must be "on-site investigation, information must be collected, information must be entered, and material evidence must be compared". The scene must be explored and all criminal cases must be investigated at the scene. Information must be collected, the site information must be collected in full time and space, not only to find and extract traditional trace evidence, but also to collect video, electronic evidence and other new evidence. Information must be recorded, and on-site investigation information and analysis must be recorded in the national geological survey system in a timely, accurate and standardized manner. Information must be compared, all traces of evidence and related information obtained from the field investigation should be matched in time.

In the current environment of criminal investigation reform, the main work is to better detect large cases (ten types of criminal cases), to detect more cases of small cases (street pickpockets, theft of non-motorized vehicles and non-contact fraud cases, etc.), more accurate cases and better control of the case. Under the requirements of the reform of the judicial system, governing the country according to law, guaranteeing judicial justice and improving the credibility of the judiciary have become the main work at present. The traditional litigation system is changing from "investigation centered" (as shown in Figure 1) to "trial centered". The principle of innocence inference, the principle of evidence referee and the 
principle of equality of accusation and defense are the three main ideas of the "trial centered" litigation system. The core idea is to fight the evidence war. Therefore, it must begin with the legitimacy of the evidence source, the legitimacy of the procedure and the integrity of the evidence chain. However, the following problems still exist in video evidence.

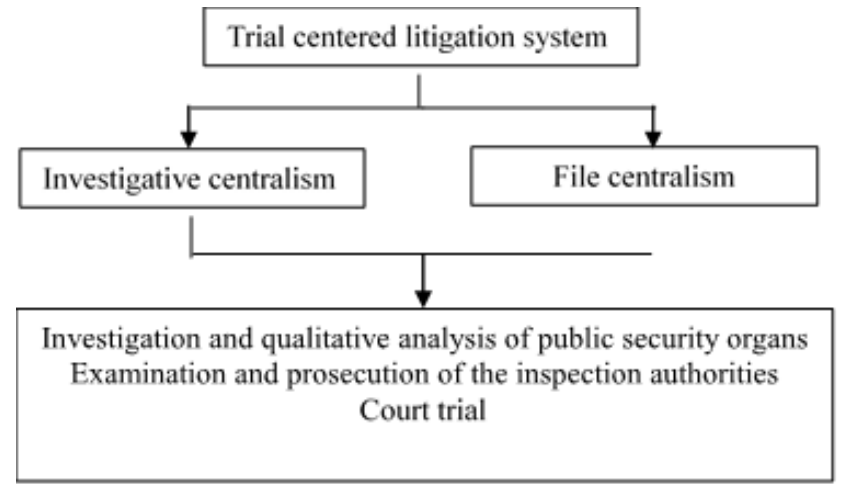

Fig. 1. The structure of a lawsuit centered on the investigation

\section{A. Video Investigation Not Adapt to the New Situation}

The investigation based on "trial center" requires a comprehensive collection of evidence. At present, the video investigation efficiency is low and many of the video sites are not explored. The quality of video investigation, the effective video evidence extraction rate and the quality of video scene investigation files are low. It can't play the role of evidence and is more difficult to face the requirements of litigation.

\section{B. The Work of Video Evidence Identification is Not Suitable}

There are some problems, such as undivided exploration, unstandardized procedures for entrustment and acceptance, confusion in the management of material evidence, disunity of inspection and identification standards, incompleteness of inspection records, and unsound work of witnesses to testify in court. These are not suited to the "trial centered" litigation evidence.

\section{Single Evidence System}

At present, the grassroots real war departments rely on the evidence of fingerprint and DNA to identify the identity of the case too much. The evidence of other related links, especially the video evidence, can't form an effective chain, which can't confirm or form evidence system with each other.

\section{DESIGN OF VIDEO STORAGE SYSTEM BASED ON CLOUD STORAGE}

At present, the public security video monitoring and storage system uses a centralized storage server to store all the data. There are many problems, such as chaos of equipment management, low efficiency of business processing, many fault nodes, insufficient capacity of massive storage, low capacity utilization and single storage application, which seriously waste the storage and computing resources of the system and can't meet the needs of storage capacity and data management. Cloud storage is the extension and development of cloud computing. By using the technology of network technology, distributed file system, server virtualization, cluster application and so on, a large number of heterogeneous storage devices in the network will eventually form a low-cost, resilient, low energy, and shared storage resource pool. A service system that provides external data storage, access and processing functions is formed.

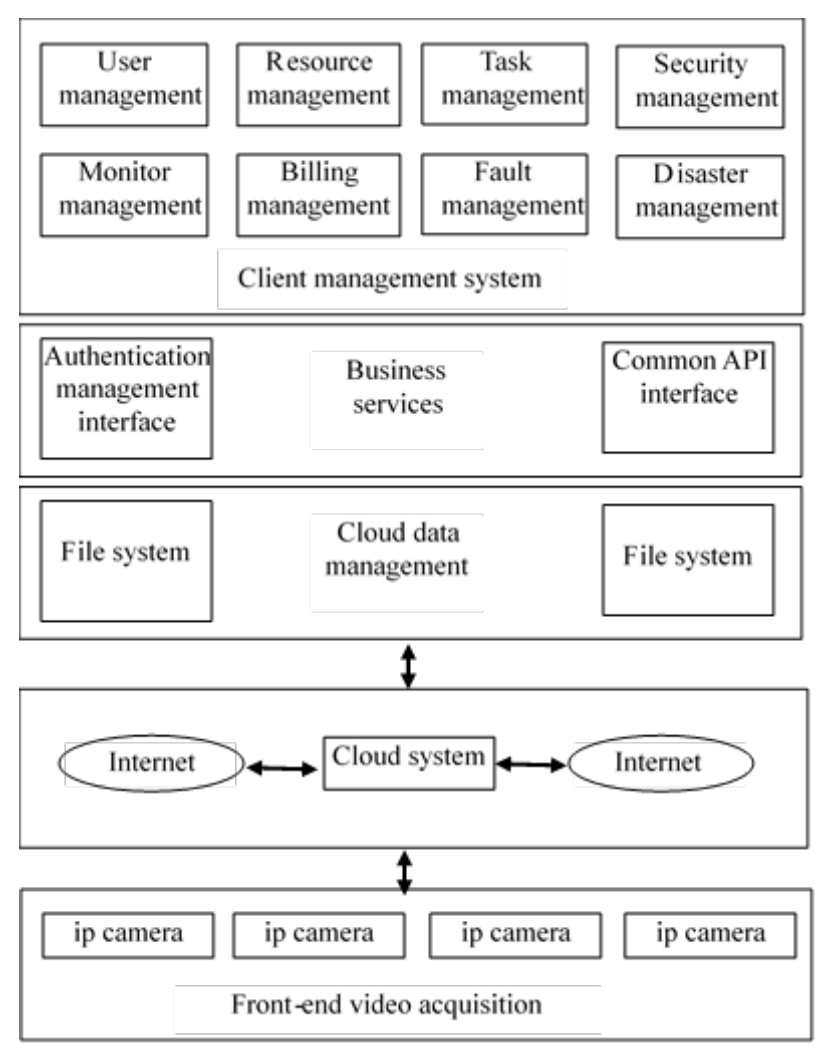

Fig. 2. Structure of video storage system based on cloud storage

This paper designs a video monitoring and storage system based on cloud storage. Video data storage and processing are deployed in the cloud by seamlessly docking existing monitoring hardware devices with video capture devices and extended devices. By analyzing the characteristics of video data and the method of data segmentation and location, the purpose of improving the utilization rate of storage space, the utilization of communication bandwidth and the optimization of data retrieval is realized. According to the actual situation, this storage system can be divided into front video acquisition, cloud data management center, video service and client management system, as shown in Figure 2.

The front end of the webcam is connected to the cloud system through the network cable and a reasonable bandwidth is set according to the transmission state of the network. Each camera first compresses the high-definition video captured, then packs it into a network-transmitted packet and uploads it to the cloud resource pool. The cloud data management center automatically monitors, real-time and centrally manage the uploaded data through the functions of virtual storage, distributed file system and cloud computing. Finally, it realizes the functions of data deletion, data compression, data encryption, data backup and data recovery. Above the cloud 
data management center is the data video service, which provides related services according to the different needs of different users. Customers access the cloud data pool through the common API interface. Only through network access, user authentication, permission settings and other security policies to verify, each business request can access the corresponding services.

\section{VIDEO EVIDENCE VOLUME COMPOSITION}

By visiting a number of front-line police and reference to the Ministry of public security's "quality standard for criminal photography"[8], the standard of monitoring video evidence volume is preliminarily formulated. This standard should be applied to the use and production of video evidence in various criminal cases, and also to the production of video evidence in public security cases (Rogue fights) and disaster accidents (aircraft crash, train subversion, traffic accident, accidental food poisoning, factory boiler explosion, etc.).

\section{A. Cover}

The cover includes: number of proof volume, title number, secret level, title of file, production organ, and time of making. There are secrets, secrets and top-secret classes among them. The name of the case should be consistent with the name of the video scene investigation record and the scene video distribution map.

\section{B. Cover Two}

Cover two mainly include: the site, the name of the case, the nature of the case, the time of the case, the video survey personnel, the volume unit of the video volume, the volume maker, the checker, the effective identification area, the total number of files, the number of images in the volume, the number of video, the number of files, and so on. The content should be accurately filled out according to the actual situation of the case.

\section{Basic Information of the Case Figures and Tables}

The basic information of the case includes the brief introduction of the case, and the emphasis should be given to the specific information such as the position of the case (latitude and longitude), the case file, the jurisdiction of the case, the time of the case, the place of the case, the case category, the time of the case, the case report person, the telephone, the instruments of crime, the offenders, the casualties and so on.

\section{Catalog}

As the content of the video evidence volume contains more content, it is necessary to write a directory, which should include the title and page number of the level of the paragraphs and the title and the page number connect by ....

\section{E. Video Volume Content}

The content part is the core of the entire video evidence volume, which should include the image involved, the related video, the lead line, the symbol, and the proper description of the text.

\section{1) Time and space information summary}

The temporal and spatial information collection mainly includes the field video distribution map of the video scene investigation (according to "Field video distribution map compilation standard" by the Ministry of Public Security), as well as the video tracks of suspects escape after the case occurred and video tracks before case occurrence.

\section{2) Material information involved in the case}

According to the video distribution map, the suspect escape video track map and the point video track chart, the suspects, the suspected vehicles and the objects in the video are captured and tagged from the video center. Finally, it clearly reflects the location of the case, the nature of the case, the process of committing a case, the means of crime, the object of the infringement, the consequences, the location and characteristics of the evidence of the evidence. All video screenshots should be organized, layered and unfolded. The size of the image, the position of the sticker and the layout of the plot refer to the standard of the quality of the criminal photography system. At the same time, indexing and writing instructions should be carried out in appropriate locations.

\section{3) Summary of video collection}

After the case, all the video related to the case need to be collected and attached to the entire evidence volume. According to the actual situation of the case, video survey information summary table includes name and location, monitoring point, calibration time, appearance time, format, credibility, cue description, ascription, extraction period and so on.

\section{MATTERS OF ATTENTION IN COLLECTION}

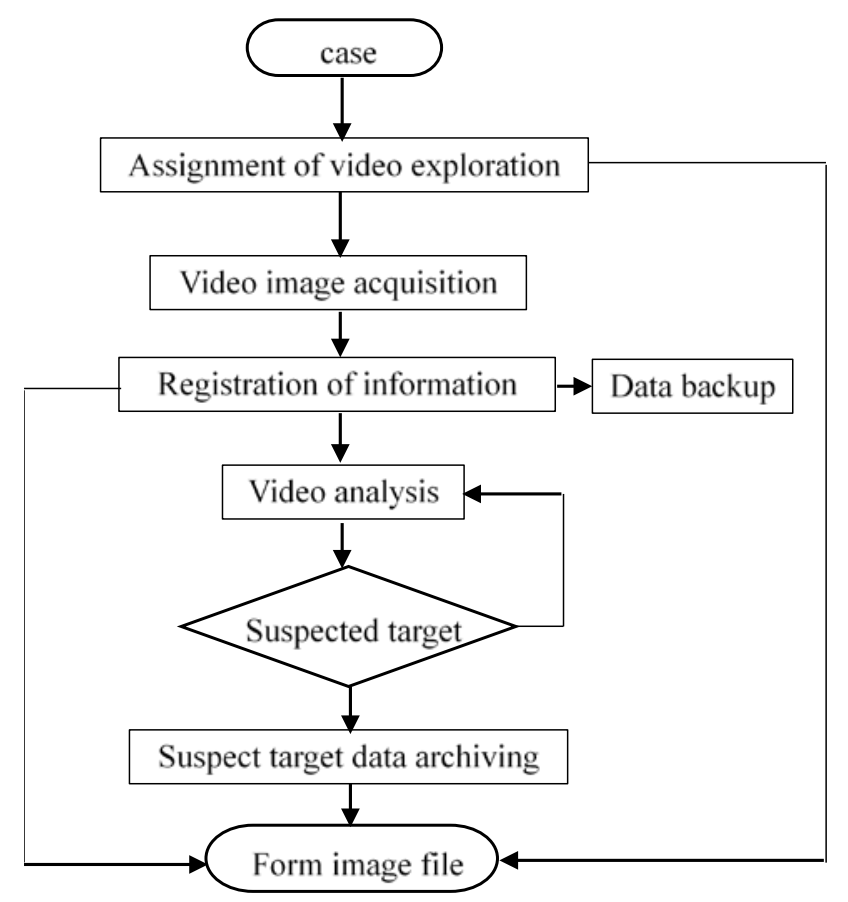

Fig. 3. Flow chart of video evidence 
Since the monitoring video has the characteristics of repeated coverage, all related video data should be quickly collected to facilitate the use of subsequent proceedings, as shown in Figure 3. Video must be collected by a formal police officer. For analog video, the number of disk, disk number, and video recording time should be recorded in detail. For digital video recording, the number of video files, number of files, size of files, and time of video recording should be recorded in detail. In order to ensure the legitimacy of the video evidence procedure, a video evidence extraction notice must be filled in and signed by the leader, the caller and the video owner. The whole process of collection should be videotaped, the collected data should be verified by MD5 code and double backup. For storage media that store important data, write protection devices are used to protect the data in the storage media and seal the data on the spot. To prevent data from being deleted and changed, we can ensure the legality and traceability of video evidence extraction.

\section{CONCLUSION}

This paper focuses on the legitimate use of surveillance video as evidence in judicial proceedings. Firstly, it introduces the current situation of video evidence in the new situation of litigation. Then a video monitoring storage system based on cloud storage is built. Finally, the standard of video evidence volume is formulated to facilitate the application of video image data to judicial proceedings by police officers. In the field of theory and application, this paper explores the feasibility of using the video data in the surveillance video as evidence in judicial proceedings and provides a new way of thinking and new methods for its further application.

\section{ACKNOWLEDGMENT}

This research work is supported by the technical research project of the Ministry of public security under contract No. 2016JSYJC59 and national key research and development plan under contract No. 2017YFC0821000.

\section{REFERENCES}

[1] Zhang Jianhua, Wu Heng, and Zhang Wenbo. "A summary of the research on core technology of cloud computing," Journal of Chinese Computer Systems, 2013(11), pp. 2417-2423.

[2] Wu Yuezhong and Zhou Xunzhi. "Analysis of core technology of cloud computing for Hadoop,” Journal of Hunan University of Technology, 2013(01), pp. 77-80.

[3] Chen Quan and Deng Qianni. “Cloud Computing and Its Key Techniques,” Journal of Computer Applications, 2009, 29(9), pp. 25622567.

[4] XU Feng. "Research on evidence based video surveillance video based on trial,” Journal of Criminal Investigation Police University of China, 2016(2), pp. 25-27.

[5] Lima $T$ and Tandayya P. "Video surveillance as a service cost estimation and pricing model,” 2015 12th International Joint Conference on Computer Science and Software Engineering, IEEE, 2015, pp. 174179.

[6] Chaisiri S, Lee B S and Niyato D. "Optimization of resource provisioning cost in cloud computing,” IEEE Transactions on Services Computing, 2012, 5(2), pp. 164-177.

[7] Joy A M. "Performance comparison between Linux containers and virtual machines," 2015 IEEE International Conference on Advances in computer Engineering and Applications (ICACEA), 2015, pp. 342-346.

[8] Zhong Zuzheng. "Research on the method of making field photography electronic files,” Legal System and Society, 2012, 8, pp. 179-180. 